\title{
In-vivo studies to manifest the potent anti- inflammatory and analgesic effect of the topical formulation, Pankajakasthuri orthoherb cream/ thermagel upon single application in wistar albino
} rats

\begin{abstract}
Pain and inflammation are the most common and prominent symptoms of many diseases. Inflammation is the most pivotal response of a damaged tissue that induces various hostile stimuli such as infections, toxic chemical agents, and physical damage resulting in redness, edema, heat, and pain. Diclofenac sodium is the most widely prescribed medicine in the management of pain and inflammation. Lamentably, long-term use of this drug causes multiple harmful side effects. To overcome this, we have formulated Pankajakasthuri orthoherb cream/thermagel, a polyherbal topical preparation for inflammation and pain relief. The objective of the present study was to assess the anti-inflammatory and analgesic potential of Pankajakasthuri orthoherb cream/thermagel, when administered topically, using carrageenan-induced edema and formalin testing in experimental animals. Here, diclofenac sodium gel was used as a positive control for anti-inflammatory and analgesic activity. From the results of our study, it was clear that Pankajakasthuri orthoherb cream thermagel significantly inhibited the formation of the rat paw edema and this effect was significantly better than standard diclofenac sodium gel. Pankajakasthuri orthoherb cream/ thermagel recorded a positive result after $3 \mathrm{~h}$ of treatment and maximum reduction in edema was recorded after $18 \mathrm{~h}$ of treatment $(96.72 \%)$. The analgesic effect of Pankajakasthuri orthoherb cream/thermagel was recorded mainly in the late phase $(70.70 \%)$. This suggests that the activity may be due to peripheral action, when compared with the action of diclofenac sodium gel. Hence it can be concluded that Pankajakasthuri orthoherb cream/ thermagel can result in better anti-inflammatory and analgesic action for a longer duration upon single application compared with standard diclofenac sodium gel.
\end{abstract}

Keywords: anti-inflammatory, analgesic, polyherbal cream, thermagel, topical application, pain
Volume 9 Issue 5 - 202I

\begin{abstract}
Hareendran Nair J, Shan Sasidharan
Department of Research and Development, Pankajakasthuri

Herbal Research Foundation, Pankajakasthuri Ayurveda Medical College Campus, India
\end{abstract}

Correspondence: Shan Sasidharan, Department of Research and Development, Pankajakasthuri Herbal Research Foundation, Pankajakasthuri Ayurveda Medical College Campus, Killy, Kattakada, Thiruvananthapuram, Kerala, India, Pin-695572, Email drshansasidharan@yahoo.co.in, drshan@pkhil.com

Received:September 10, 2021 | Published: September 22, 2021

\section{Introduction}

Inflammation in humans is mainly depicted by pain, redness, swelling and dysfunction of the tissues and organs. It is a common outcome of the host's protective responses to tissue injury induced by a variety of stimuli (for example, physical trauma, toxins, and infectious agents). ${ }^{1,2}$ Inflammatory disorders, such as arthritis, cardiovascular disease, diabetes mellitus, and others, have become major public health concerns in recent years, leading to an increase in annual mortality rates. ${ }^{3}$ Due to the release of algesic mediators, inflammation is frequently associated with pain as a subsequent event. ${ }^{4}$

Pain is the most common symptom used to diagnose a variety of diseases, and it is widely accepted as one of the most important predictors of quality of life. Most conventional pain medications are now unsatisfactory in efficacy, tolerability, and toxicity. ${ }^{5}$ Despite recent advances in therapy, there is still a need for effective and potent analgesic and anti-inflammatory drugs.

Inflammation is a hallmark of several chronic diseases and the topical drugs mainly used for skin inflammation treatment are corticosteroids and nonsteroidal anti-inflammatory drugs (NSAIDs) ${ }^{6}$ However, the routine treatment of inflammatory disease with these agents over long periods leads to adverse effects, including pruritus, irritations, skin dryness, folliculitis, hypertrichosis, acneiform eruptions, hypopigmentation, allergic contact dermatitis, skin maceration, stretch marks and military. ${ }^{7,8}$ Therefore, research to identify anti-inflammatory and analgesic compounds with fewer side effects and more efficacies is necessary. Complementary to this, herbal blends are one of the most imperative sources for discovering new drugs with a high safety margin.

In recent times Pankajakasthuri Herbals India Pvt. Ltd. has formulated Pankajakasthuri orthoherb cream/thermagel (DL. NO: $50 / 25 \mathrm{D} / 96$ ) to manage the severe inflammation and joint pain. This product contains 21 active Ayurvedic ingredients like Adathoda vasica, Aegle marmelos, Ricinus communis, Azadirecta indica, Sida retusa, Tragia involucrata, etc., along with essential oils that could increase blood circulation/blood pooling through vasodilation and could relieve pain through counter stimulation. Several research articles were available on the anti-inflammatory and analgesic properties of the ingredients used for the formulation of Pankajakasthuri orthoherb cream/thermagel. ${ }^{9-18}$ The herbal ingredients used for preparation are characterized by abundance in bioactive phytochemicals, like polyphenols, carotenoids, flavonoids, lignans, stillbenoids, phytosterols and essential oil substances possessing significant anti- 
inflammatory and analgesic activity. ${ }^{19,20}$ The specialty of orthoherb cream/thermagel is that it could provide the same result as applying a traditional Ayurvedic kizhi (bolus/poultice), which is generally prescribed treatment of problems related to the muscles, bones and joints. The warmth of the poultices, combined with the active ingredients of the herbs and oils, improves circulation and reduces body stiffness and pain.

However, advanced studies are needed to introduce Pankajakasthuri orthoherb cream/thermagel for its widespread clinical application. This will not only confirm its effectiveness but also enable its acceptance in treating the target illness. Therefore, the present work was carried out only to assess the anti-inflammatory and analgesic activity of orthoherb cream/thermagel in the experimental animal model upon single application and further compare its activity with the standard drug (diclofenac).

\section{Materials and methods}

\section{Chemicals}

Diclofenac sodium used in this study was purchased from SigmaAldrich (St. Louis, MO, USA). Formalin was purchased from Merck (Germany). Carrageenan was purchased from HiMedia, Mumbai, India.

\section{Test drug (Pankajakasthuri orthoherb cream/ thermagel)}

The test drug is Pankajakasthuri orthoherb cream/thermagel, an herbal-based cream/thermagel preparation for topical administration. Pankajakasthuri orthoherb cream/thermagel is manufactured at Good Manufacturing Practices approved production line at Pankajakasthuri Herbals India Pvt. Ltd. situated at Poovachal, Kattakada, Thiruvananthapuram, Kerala, India.

\section{Preparation of standard drug (Diclofenac sodium gel)}

The diclofenac sodium was used as the standard drug in this study. For animal experiments, diclofenac sodium gel was prepared extemporarily by mixing diclofenac sodium $(3 \% \mathrm{w} / \mathrm{w})$ in pre-weighted amounts of gel. After preparation, the gel was filled in collapsible tubes and stored in cool, dry place.

\section{Experimental animals}

In this study, adult Wistar albino rats weighing 150-200 g were used. The rats were maintained in standard laboratory conditions $\left(22^{\circ} \mathrm{C}\right.$ room temperature and $65-70 \%$ humidity) with $12 \mathrm{~h}$ light and dark cycles and were fed and watered (ad libitum). Prior to the experiment, the rats were fed pellet food and acclimatized to laboratory conditions for 7 days. The Institutional Animal Ethics Committee of CARe KERALAM granted approval for conducting this work (Approval No. CKL/TOX/IAEC/2021-2/158). All the animal experimental protocols were in accordance with the guidelines of the Committee for the Purpose of Control and Supervision of Experiments on Animals (CPCSEA), Ministry of Forest and Environment, Government of India.

\section{Determination of anti-inflammatory study of Pankajakasthuri orthoherb cream/thermagel}

\section{Carrageenan-induced rat paw edema}

The anti-inflammatory activity was evaluated using carrageenan induced paw edema. Initially, animals were divided into three groups $(\mathrm{n}=6)$ and were treated as mentioned in Table 1. Carrageenan $(50 \mu 1$ of $1 \%$ carrageenan in normal saline,) was injected into the plantar surface of the rats right hind paw to produce acute inflammation. For checking the anti-inflammatory activity, $0.3 \mathrm{~g}$ of each treatment gel (plain cream/gel base, Pankajakasthuri orthoherb cream/thermagel and diclofenac sodium gel) was applied to the surface of the hind paw and massaged gently 50 times with the index finger. Rats of the control groups received only the cream base. Diclofenac sodium gel was used as standard drug. The test drug, cream/gel base and standard drug were applied $30 \mathrm{~min}$ prior to injection of carrageenan (sub plantar). The paw thickness $(\mathrm{mm})$ was measured immediately after carrageenan injection and at $0.5,1,2,2.5,3,4,6,12,18$ and $24 \mathrm{~h}$ intervals after the administration of the samples using digital Vernier caliper (INSIZE, DVC-01). ${ }^{21-26}$ The calculations were performed using the following formulas.

Table I Treatment of animals in Carrageenan-induced rat paw edema model

\begin{tabular}{llll}
\hline S. No & Groups & $\begin{array}{l}\text { No. of } \\
\text { animals }\end{array}$ & Treatment \\
\hline I & Control (Plain cream base) & 6 & Carrageenan $(0.1 \mathrm{ml}$ of I\% solution, subplantar inj., left hind paw) \\
2 & Test (Orthoherb cream/thermagel) & 6 & $\begin{array}{l}\text { Carrageenan }(0.1 \mathrm{ml} \text { of I\% solution, subplantar inj., left hind paw })+0.3 \mathrm{~g} \\
\text { Orthoherb cream, topically }\end{array}$ \\
3 & Standard & 6 & $\begin{array}{l}\text { Carrageenan }(0.1 \mathrm{ml} \text { of I\% solution, subplantar inj., left hind paw })+0.3 \mathrm{~g} \\
\text { Diclofenac gel topically }\end{array}$
\end{tabular}

Change in paw Edema $(\Delta \mathrm{T})$ was calculated as follows:

$\Delta \mathrm{T}=\mathrm{Tt}-\mathrm{T} 0$

Percentages reduction of paw thickness was obtained using the following formula:

$$
\frac{(T t-T 0) \text { control }-(T t-T 0) \text { treated } \times 100}{(T t-T 0) \text { control }}
$$

Where $\mathrm{Tt}$ is the hind paw thickness $(\mathrm{mm})$ at time ' $\mathrm{t}$ ', $\mathrm{T} 0$ is hind paw thickness $(\mathrm{mm})$ before sub plantar injection.

\section{Determination of analgesic activity of Pankajakasthuri orthoherb cream/thermagel}

\section{Formalin test}

The analgesic activity was conducted according to the method of Dubuisson and Dennis ${ }^{27}$ with minor modification. In this experiment, animals were divided into three groups $(n=6)$ and the treatments were performed as mentioned in Table 2. On the plantar surface of the right hind paw, $0.3 \mathrm{~g}$ of treatment gel was applied and gently rubbing 50 times with the index finger. The cream base was given to the rats in 
the control groups. Diclofenac sodium gel applied in the same way was used as reference. Fifteen minutes later, the antinociceptive activity was determined. Fifty microliters of $5 \%$ formalin was injected subcutaneously on the dorsal surface of the right fore paw. The rats were observed for $60 \mathrm{~min}$ after the formalin injection, and the amount of time spent licking the injected hind paw was recorded. The early phase is defined as the first 5 minutes after formalin injection, and the late phase is defined as the time between 15 and 60 minutes. Each rat behavior was rated separately according to the scale detailed in Table 3.

Table 2 Treatment of animals in Formalin test

\begin{tabular}{llll}
\hline S.No & Groups & No. of animals & Treatment \\
\hline I & Formalin control & 6 & Formalin $(50 \mu \mathrm{l}$ of $5 \%$ solution, subcutaneous inj., right fore paw $)$ \\
2 & Test & 6 & $\begin{array}{l}\text { Formalin }(50 \mu \mathrm{l} \text { of } 5 \% \text { solution, subcutaneous inj., right fore paw })+ \\
0.3 \mathrm{~g} \text { Orthoherb cream, topically }\end{array}$ \\
3 & Standard & 6 & $\begin{array}{l}\text { Formalin }(50 \mu \mathrm{l} \text { of } 5 \% \text { solution, subcutaneous inj., right fore paw })+ \\
0.3 \mathrm{~g} \text { Diclofenac gel topically }\end{array}$ \\
\hline
\end{tabular}

Table 3 Pain rating scale

Score Animal Behavior
Both forepaws are placed on the floor, and weight is evenly distributed. During locomotion, there is no discernible favoring of the
injected paw.
The injected paw rests lightly on the floor or another part of the animal's body, and little or no weight is placed upon it. During
locomotion, there is an obvious limp. This rating is also given to normal grooming, during which both paws are elevated and "washed",
and to rearing where neither forepaw is in contact with the floor, although either may be in contact with the wall.Attempts to sleep by
curling up with both paws off the floor are also given this rating.
The injected paw is elevated, and not in contact with any surface. The uninjected paw is placed firmly on the floor.Attempts to sleep by
curling up with only the injected paw off the floor, even when tucked under the body, are given this rating.
The injected paw is licked, bitten, or shaken, while the uninjected paw is not. This behavior is quite distinct from normal grooming (rating
I), although transitions between the two are common.

\section{Statistical analysis}

All the results were expressed as mean \pm SEM. One-way ANOVA with Dunnets post-test was used to analyze the significance of the results. Statistical significance set at $P, 0.05$. All statistical analyses were performed using GraphPad Prism, version 5.0.

\section{Results}

This study revealed that the topical application of Pankajakasthuri orthoherb cream/thermagel has remarkable anti-inflammatory action of hind paw edema in rats induced by carrageenan. In addition to this, the drug appreciably exposed its analgesic activity by inhibiting the formalin-induced nociception. The results are shown in Tables 4-5 and Figures 1-3.

Table 4 Effect of topical administration of Orthoherb cream/thermagel on paw thickness in carrageenan-injected rats

\begin{tabular}{|c|c|c|c|c|c|c|c|c|c|c|c|}
\hline \multirow{2}{*}{ Group } & \multicolumn{11}{|c|}{ Paw thickness $(\mathrm{mm})$ at time after carrageenan injection } \\
\hline & Initial & $0.5 \mathrm{~h}$ & I h & $2 \mathrm{~h}$ & $2.5 \mathrm{~h}$ & $3 \mathrm{~h}$ & $4 \mathrm{~h}$ & $6 \mathrm{~h}$ & $12 \mathrm{~h}$ & $18 \mathrm{~h}$ & $24 \mathrm{~h}$ \\
\hline Control & $3.50 \pm 0.09$ & $4.51 \pm 0.09$ & $4.85 \pm 0.06$ & $5.16 \pm 0.13$ & $4.93 \pm 0.08$ & $4.91 \pm 0.08$ & $5.05 \pm 0.07$ & $4.7 I \pm 0.09$ & $4.61 \pm 0.09$ & $4.47 \pm 0.09$ & 4. $10 \pm 0.10$ \\
\hline Test & $3.47 \pm 0.13$ & $4.52 \pm 0.15$ & $4.87 \pm 0.22$ & $5.14 \pm 0.17$ & $4.66 \pm 0.16$ & $4.34 \pm 0.16 * *$ & $3.95 \pm 0.18^{* * *}$ & $3.67 \pm 0.15^{\text {**** }}$ & $3.59 \pm 0.14 * * *$ & $3.50 \pm 0.13^{* * *}$ & $3.49 \pm 0.13^{* *}$ \\
\hline \multicolumn{12}{|c|}{ Percentage of inhibition } \\
\hline & & $(-4.82)$ & $(-4.22)$ & $(-0.60)$ & -16.18 & -37.69 & -68.68 & -84.35 & -88.96 & -96.72 & -96.1 \\
\hline Standard & $3.18 \pm 0.09$ & $4.02 \pm 0.09$ & $4.40 \pm 0.09$ & $4.46 \pm 0.13 * *$ & $3.73 \pm 0.10^{* * * *}$ & $3.69 \pm 0.09 * * *$ & $3.65 \pm 0.09 * * *$ & $3.59 \pm 0.09 * * *$ & $3.56 \pm 0.09 * * *$ & $3.53 \pm 0.09 * * *$ & $3.50 \pm 0.08^{* *}$ \\
\hline \multicolumn{12}{|c|}{ Percentage of inhibition } \\
\hline & & -16.28 & -8.81 & -22.51 & -60.84 & -63.38 & -69.65 & -66.07 & -64.9 & -62.93 & -45.68 \\
\hline
\end{tabular}

Values are mean \pm S.E.M. (percent reduction)

${ }^{*} p<0.05,{ }^{* *} p<0.01$, ${ }^{* * * *} p<0.001$

Citation: Nair HJ, Sasidharan S. In-vivo studies to manifest the potent anti-inflammatory and analgesic effect of the topical formulation, Pankajakasthuri orthoherb cream/thermagel upon single application in wistar albino rats. Pharm Pharmacol Int J. 202 I;9(5):20I-208. DOI: I0.15406/ppij.202I.09.00345 
Table 5 Effect of orthoherb cream/thermagel on formalin-induced edema in rats

\begin{tabular}{|c|c|c|c|c|c|c|c|}
\hline \multirow{2}{*}{ S. No } & \multirow{2}{*}{ Group } & \multicolumn{2}{|c|}{ Licking time (s) } & \multicolumn{2}{|l|}{ Pain score } & \multicolumn{2}{|c|}{$\%$ of inhibition } \\
\hline & & Phase I & Phase II & Phase I & Phase II & Phase I & Phase II \\
\hline 1 & Control & $74.17 \pm 1.35$ & $95.00 \pm 6.49$ & $2 .|7 \pm 0.4|$ & $1.00 \pm 0.00$ & - & - \\
\hline 2 & Test & $39.83 \pm 0.79 * * *$ & $27.83 \pm 2.20 * * *$ & $1.00 \pm 0.00 * * *$ & $0.17 \pm 0.4 I^{* *}$ & 46.29 & 70.7 \\
\hline 3 & Standard & $41.33 \pm 1.52 * * *$ & $29.00 \pm 2.57 * * *$ & $1.00 \pm 0.00 * * *$ & $0.17 \pm 0.4 I^{* *}$ & 44.27 & 69.47 \\
\hline
\end{tabular}

Values are mean \pm S.E.M

$*_{\mathrm{p}}<0.05, * *_{\mathrm{p}}<0.01, * * *_{\mathrm{p}}<0.001$

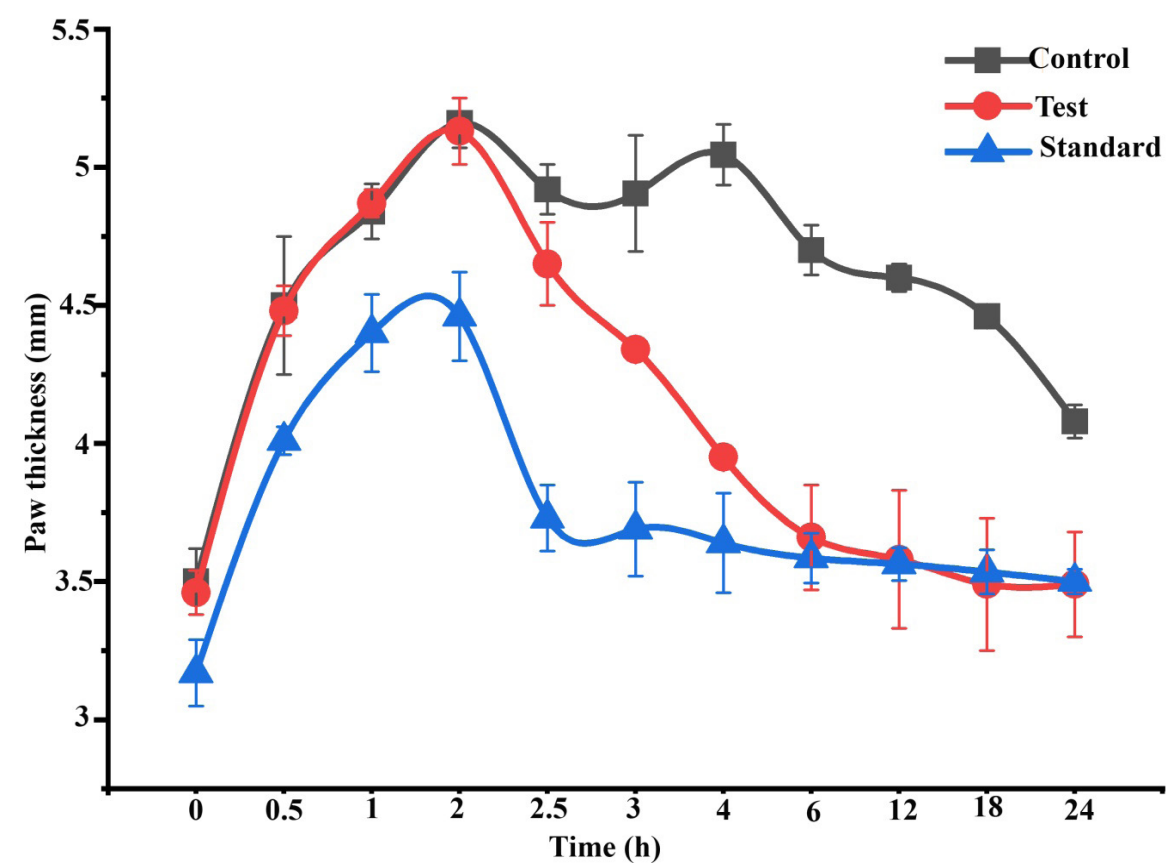

Figure I Effect of topical administration of orthoherb cream/thermagel on paw thickness in carrageenan-injected rats. Data represented as mean \pm S.E.M ( $n=6$ ).

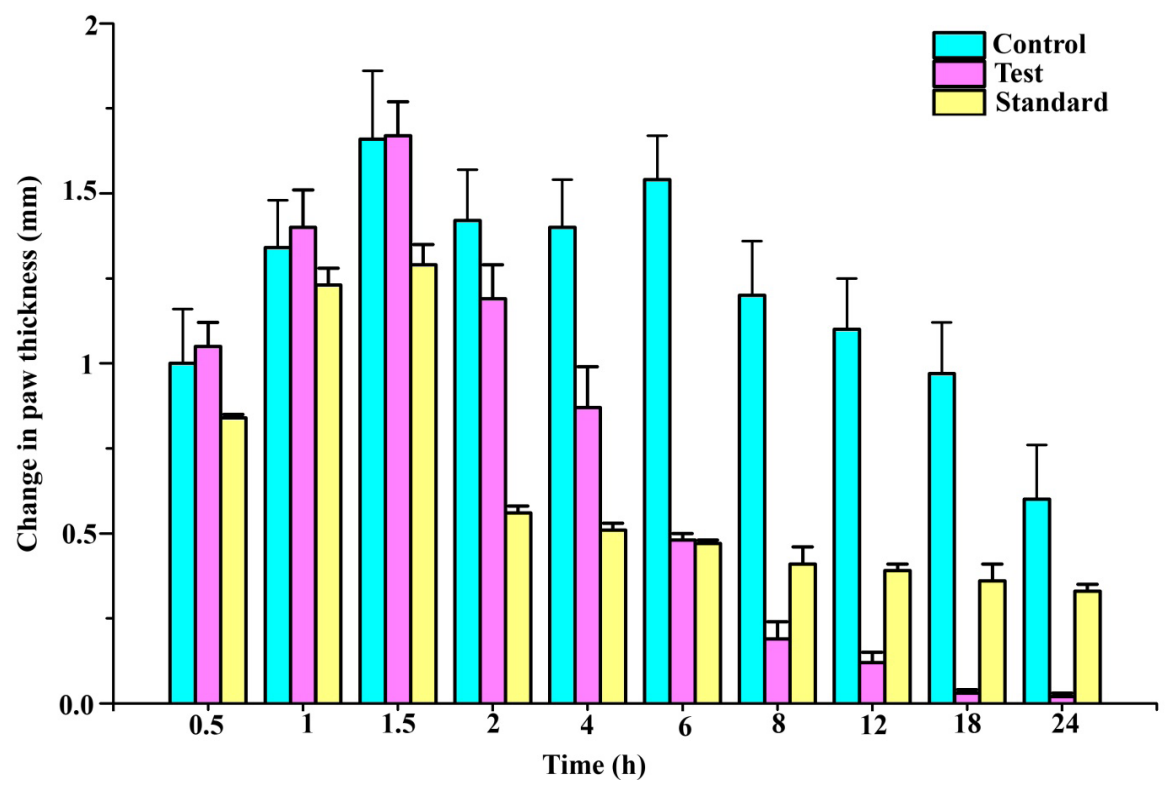

Figure 2 Effect of topical administration of Orthoherb cream/thermagel on change in paw edema in carrageenan-injected rats. Data represented as mean \pm S.E.M $(n=6)$. 


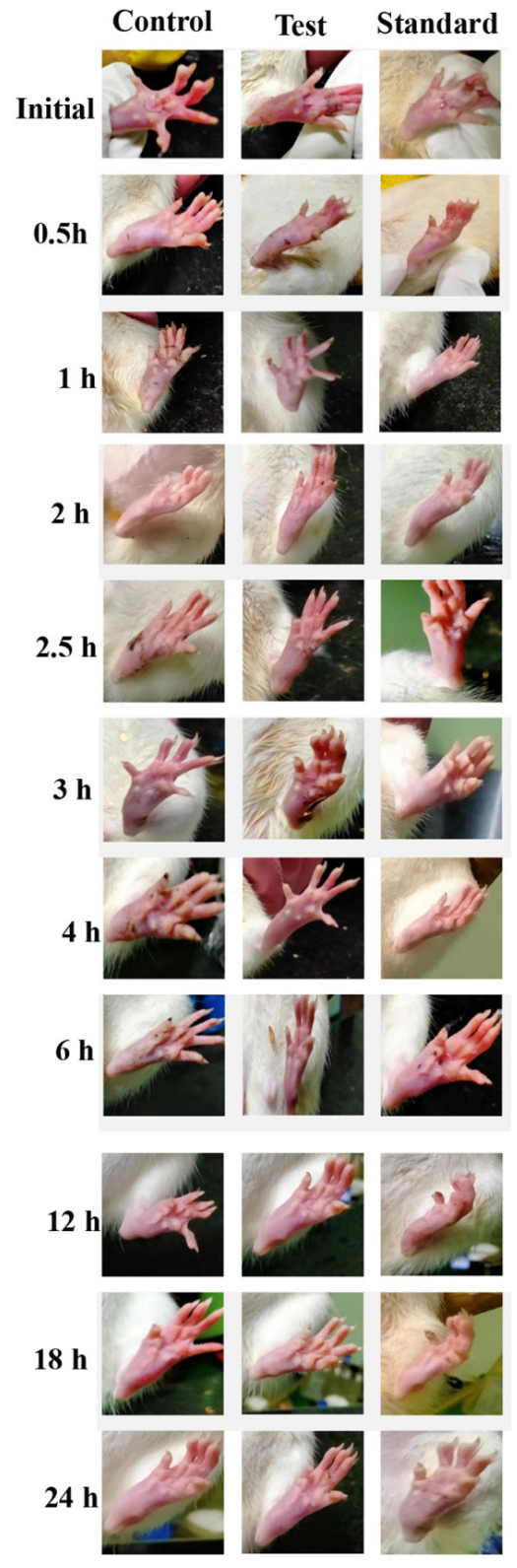

Figure 3 Gross appearance of paw in carrageenan-injected rats of three groups at different time points.

Sub plantar injection of carrageenan in rats resulted in timedependent inflation in paw thickness (Figure 1). This inflation was observed up to $4 \mathrm{~h}$ after administration of carrageenan injection in the control groups, which was treated with plain cream/gel base. Pankajakasthuri orthoherb cream/thermagel induced a time-dependent reduction in paw edema. In the case of Pankajakasthuri orthoherb cream/thermagel, the significant anti-inflammatory action $(p<0.01)$ was recorded $3 \mathrm{~h}$ after carrageenan injection and a most effective edema reduction $(p<0.001)$ was recorded between 4 to $18 \mathrm{~h}$. The maximum percent reduction $(96.72 \%)$ in edema by Pankajakasthuri orthoherb cream/thermagel was recorded at $18 \mathrm{~h}$ after carrageenan injection. The thickness of paw at $24 \mathrm{~h}(3.49 \pm 0.13)$ in Pankajakasthuri orthoherb cream/thermagel treated animals was found very much

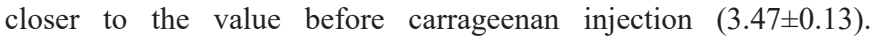
However, carrageenan-induced inflammation was reduced in all phases of the experiment by applying diclofenac gel (standard drug), with a significant reduction $(\mathrm{p}<0.01)$ starting at $2 \mathrm{~h}$ after injecting carrageenan. Diclofenac gel resulted in maximum edema reduction $(69.65 \%)$ at $4 \mathrm{~h}$ and subsequently, it showed a decreasing tendency up to $24 \mathrm{~h}(45.68 \%)$. There was no significant difference in change in paw thickness at $24 \mathrm{~h}$ compared to control. However, at $24 \mathrm{~h}$, the change in paw thickness was significantly reduced $(\mathrm{p}<0.001)$ in the Pankajakasthuri orthoherb cream/thermagel treated animals $(0.02 \pm 0.01)$ compared to the control $(0.60 \pm 0.16)$ and standard drug $(0.33 \pm 0.02)$ treated animals.

The effects of orthoherb cream/thermagel on formalin test are shown in Table 5. In the early phase i.e. Phase I, Pankajakasthuri orthoherb cream/thermagel and diclofenac sodium gel (standard drug) significantly $(P<0.001)$ inhibited the licking response compared with the control group, with percentages of $46.29 \%$ and $44.27 \%$, respectively. In the late phase (Phase II), Pankajakasthuri orthoherb cream/thermagel and diclofenac exerted significant $(P<0.001)$ and the maximum inhibitory effects on the licking response $(70.70 \%$ and $69.47 \%$, respectively). Hence, it is evident that a marked reduction in licking time was observed in rats treated with Pankajakasthuri orthoherb cream/thermagel as well as with diclofenac gel compared to the control group animals on both early and late phases. The pain rating score of formalin-induced behavior indicated that Pankajakasthuri orthoherb cream/thermagel produced a significant analgesic effect in both the phases of pain and was found to be comparable to diclofenac in attenuating pain in this model upon single application.

\section{Discussion}

Humans are continually racing to get ahead of one another in today's competitive society. As a result, we jeopardize our health and experience suffering, such as headaches and back pain. Many of these aches and pains are caused by our way of life and occupational hazards. Pain and inflammation are the main symptoms in a great variety of pathological states, both acute, e.g., trauma due to sprains, strains and bruises, as well as chronical, such as chronic venous insufficiency, osteoarthritis of superficial joints and localized forms of soft tissue rheumatism. We use pain killers to help us cope with the pain. Herbs are considered to be a significant source of potentially beneficial constituents for the development of new therapeutic agents, as most of them are safe with fewer or no side effects. Alternative therapies for the treatment of inflammation are still being developed around the world, as many of the already available analgesics and antiinflammatory pharmaceuticals have various side effects, such as gastro-intestinal ulcers, bleeding, renal disorders, and so on. Nowadays, herbal gels are becoming popular due to their safe and effective use. ${ }^{28}$ This research aimed to investigate the anti-inflammatory and analgesic properties of Pankajakasthuri orthoherb cream/thermagel upon single application to identify an effective natural alternative for the treatment of the symptoms mentioned above. Pankajakasthuri orthoherb cream/ thermagel is a polyherbal formulation in cream/gel form formulated using medicinal plants that were reported to have a good effect in treating pain and inflammation as per the previous studies. ${ }^{9-18}$ The finding of our study clearly revealed that the topical application of Pankajakasthuri orthoherb cream/thermagel markedly suppressed the hind paw edema induced by carrageenan and inhibited the formalininduced nociception upon single application.

The anti-inflammatory activity of Pankajakasthuri orthoherb cream/ thermagel was studied using the carrageenan induced rat paw edema method. The effect of this polyherbal gel was compared with that of standard diclofenac gel (3\%) on rat paw edema. Carrageenan-induced paw edema is a reproducible model that can be extensively utilized 
to evaluate novel compounds/formulations for anti-inflammatory activity. Carrageenan resulted in acute inflammation and it is a wellestablished model to assess the anti-inflammatory effect of the natural products. ${ }^{29}$ Development of edema in the rat paw after carrageenan injection is demonstrated to be a biphasic effect. ${ }^{30}$ The initial phase occurring during the first hour is due to the release of histamine and serotonin. ${ }^{31}$ The second phase of edema is attributed to the release of prostaglandins, protease and lysosome. ${ }^{30,31}$ Based on this, it could be stated that the suppression of the first phase of edema may be due to inhibition of the release of early mediators, like histamine and serotonin, and the edema reduction in the second phase may be caused by inhibition of cyclooxygenase. The present study indicates that Pankajakasthuri orthoherb cream/thermagel significantly inhibited the formation of rat paw edema in the late phase. This effect is similar to that produced by non-steroidal anti-inflammatory drugs like diclofenac sodium. Pankajakasthuri orthoherb cream/thermagel has recorded its positive effect after $3 \mathrm{~h}$ of carrageenan treatment and a maximum edema inhibition $(96.72 \%)$ was observed after $18 \mathrm{~h}$ of treatment. There observed a near normalization of the paw thickness by $24 \mathrm{~h}$ after carrageenan injection in orthoherb treated animals. It can be perceived that the Pankajakasthuri orthoherb cream/thermagel also prolonged the edema reduction up to $18 \mathrm{~h}$ to $24 \mathrm{~h}$ after the carrageenan injection, unlike Diclofenac gel, which resulted in maximum edema inhibition up to $4 \mathrm{~h}$ only. Hence it can be pointed that orthoherb cream/ thermagel can result in prolonged anti-inflammatory effects upon a single application. So, it can be perceived that the anti-inflammatory effect of orthoherb cream/thermagel might be significantly better than the standard diclofenac gel in managing the inflammations.

Nonsteroidal anti-inflammatory medicines have also been demonstrated to inhibit mediators such serotonin, bradykinin, and capsaicin, ${ }^{32}$ all of which have been linked to carrageenan-induced paw oedema. It's not surprising that diclofenac reduced carrageenaninduced oedema in the right hind paw of rats in this study. Pankajakasthuri orthoherb cream/thermagel significantly reduced carrageenan-induced oedema in the right hind paw of rats, implying that the test drug is likely impacting a variety of mediators to create its anti-inflammatory action.

In this study, the analgesic effect of the Pankajakasthuri orthoherb cream/thermagel was evaluated using the formalin test in the rat. The formalin test is a highly reproducible and common in vivo model for the evaluation of the analgesic potential of drugs and possible mechanisms. Formalin test induced a distinct biphasic response and various analgesics may act differently in the early and late phases of this test. Therefore, the test can be used to define the possible mechanism of the antinociceptive effect of a proposed analgesic. ${ }^{33}$ Centrally-acting drugs like opioids inhibit both phases equally, ${ }^{34}$ but peripherally acting drugs namely aspirin, indomethacin, diclofenac sodium and dexamethasone inhibit the late phase. The late phase is considered to be anti-inflammatory response with inflammatory pain that can be suppressed by anti-inflammatory drugs. ${ }^{35,36}$ The highest activity of Pankajakasthuri orthoherb cream/thermagel was recorded on the late phase of formalin test suggests that its activity may be due to peripheral action, when compared with diclofenac gel activity in this respect. Based on the results from the present study, it can be suggested that Pankajakasthuri orthoherb cream/thermagel possess potent analgesic action and this analgesic effect may be attributed to inhibition of prostaglandin release and other mediators involved in this test.

Previous studies conducted on dermal irritation potential of Pankajakasthuri orthoherb cream/thermagel via, single and repeated exposure, revealed that this formulation was non-irritant or produced negligible irritation on rabbit skin following a 14 day observational period and hence regarded as safe for topical application. From these results, we can finally state that the topical polyherbal preparation named Pankajakasthuri orthoherb cream/thermagel might possess an anti-inflammatory and analgesic effect, which can be useful for the safe treatment of local inflammation. Phytochemical investigations of herbs like Adathoda vasica, Aegle marmelos, Ricinus communis, Azadirecta indica, Sida retusa, Tragia involucrata., which are the major medicinal herbs included in the Orthoherb cream/thermagel, revealed the presence of flavonoid compounds, alkaloids, steroids, tannins, saponins etc. ${ }^{37,42}$ Flavonoids have been exhibited to hold various biological properties related to antioxidant, antinociceptive, and anti-inflammatory mechanisms by targeting reactive oxygen species and prostaglandins which are associated in the late phase of acute inflammation and pain perception. ${ }^{43-45}$ Also, alkaloids and glycosides are compounds known for their anti-inflammatory activity. ${ }^{46}$ Therefore, it is possible that both the anti-nociceptive and anti-inflammatory effects noted with Pankajakasthuri orthoherb cream/thermagel may be attributable to them. However, further studies are necessary to elucidate the exact mechanism of action of this polyherbal formulation.

\section{Conclusion}

The pharmacological tests performed in the present study confirmed the analgesic and anti-inflammatory activity of Pankajakasthuri orthoherb cream/thermagel and revealed that it possessed a varying degree of central, peripheral analgesic and acute anti-inflammatory activity that could be attributed to the presence of various bioactive agents including flavonoids, tannins, terpenoids, saponins, steroids, glycosides, alkaloids and essential oils that might have acted synergistically. Furthermore, the data obtained in this study also demonstrated that Pankajakasthuri orthoherb cream/thermagel is an influential and effective topical formulation and it recorded better anti-inflammatory and analgesic activity compared with standard diclofenac sodium gel. This study also supports the potential of various herbs, incorporated in this formulation, for its promising effects in the treatment of pain and inflammation.

\section{Major outcome of the study}

Pankajakasthuri orthoherb cream/thermagel demonstrated to have the following outcomes:

- Potent and prolonged anti-inflammatory effect upon single application

- This resulted in normalization of the rat paw thickness by $24 \mathrm{~h}$ after carrageenan injection.

- Potent analgesic action.

- Possible mechanism of actions including inhibition of cyclooxygenase and prostaglandin release.

- Better anti-inflammatory and analgesic action for longer duration upon single application compared with standard diclofenac sodium gel

- Recommended dosing schedule-once/twice daily for prolonged anti-inflammatory and analgesic effect.

\section{Acknowledgments}

Authors give adored Pranams to "Aadhyathma Chinthalayesan", Chinthala Ashram, Pothencode, Trivandrum, Kerala, India for his benevolence and blessings. We sincerely acknowledge 
Pankajakasthuri Herbal Research Foundation, Kattakada, Thiruvananthapuram, Kerala, India and Pankajakasthuri Herbals India Pvt. Ltd Poovachal, Kattakada, Thiruvananthapuram, Kerala, India for providing necessary support to conduct studies. Further, we thank all the Directors and staffs of Pankajakasthuri Herbal Research Foundation and Pankajakasthuri Herbals India Pvt. Ltd for their supports to complete this work successfully. The authors sincerely thank The Director, CARe KERALA, Koratty, Thrissur, Kerala, India for providing the necessary infrastructure for carrying the animal studies.

\section{Conflicts of interest}

Authors declare that there is no conflict of interest.

\section{References}

1. Ehlers S, Kaufmann SH. Infection, inflammation, and chronic diseases: consequences of a modern lifestyle. Trends Immunol. 2010;31(5):184 190 .

2. Szatmari SZ, Whitehouse PJ. Vinpocetine for cognitive impairment and dementia. Cochrane database Syst Rev. 2003;2003(1):CD003119.

3. Gou KJ, Zeng R, Dong Y, et al. Anti-inflammatory and Analgesic Effects of Polygonum orientale L. Extracts. Front Pharmacol. 2017;8:562.

4. Cruz MP, Andrade CMF, Silva KO, et al. Antinoceptive and Antiinflammatory Activities of the Ethanolic Extract, Fractions and Flavones Isolated from Mimosa tenuiflora (Willd.) Poir (Leguminosae). PLoS One. 2016;11(3):e0150839.

5. Scholz J, Woolf CJ. Can we conquer pain? Nat Rev Neurosci. 2002;5 Suppl:1062-1067.

6. Selvarani K, Bai G. Anti-arthritic activity of Cayratia pedata leaf extract in Freund's adjuvant induced arthritic rats. Semantic scholar; 2014

7. Gomes S, Bhattacharya M, Chakraborty P, et al. Anti-arthritic activity of Indian monocellate cobra (Naja kaouthia) venom on adjuvante induced arthritis. Toxicon. 2010;55(2):670-673.

8. Kanagasanthosh K, Shanmugapriyan S, Kavirajan V. Evaluation of acute toxicity, anti-inflammatory activity and phytochemical screening of ethanolic extract of Azadirachta indica leaves. Int J Res Dev Pharm. 2015;4(5):1737-1742.

9. Mulla W, More S, Jamge SB et al. Evaluation of anti-inflammatory and analgesic activities of ethanolic extract of roots Adhatoda vasica Linn. International Journal of Pharm Tech Research. 2010;2:1364-1368.

10. Sanjay K, Udaya KV, Agrawal D et al. Analgesic, Anti-inflammatory and Anti-Pyretic Effects of Azadirachta indica (Neem) Leaf Extract in Albino Rats. International Journal of Science and Research. 2015;4(8):713-721.

11. Naik MR, Bhattacharya A, Behera R, et al. Study of anti-inflammatory effect of neem seed oil (Azadirachta indica) on infected albino rats. $J$ Health Res Rev. 2014;1(3):66-69.

12. Arul V, Miyazaki S, Dhananjayan R. Studies on the anti-inflammatory, antipyretic and analgesic properties of the leaves of Aegle marmelos Corr. J Ethnopharmacol. 2005;96(1-2):159-163.

13. Benni JM, Jayanthi MK, Suresha RN. Evaluation of the anti-inflammatory activity of Aegle marmelos (Bilwa) root. Indian Journal of Pharmacology. 2011;43(4):393-397.

14. Ilavarasan R, Mallika M, Venkataraman S. Anti-inflammatory and free radical scavenging activity of Ricinus communis root extract. $J$ Ethnopharmacol. 2006;103(3):478-480.

15. Taur DJ, Waghmare MG, Bandal RS et al. Antinociceptive activity of Ricinus communis L. leaves. Asian Pacific journal of tropical biomedicine. 2011;1(2):139-141
16. Franzotti EM, Santos CV, Rodrigues HM et al. Anti-inflammatory, analgesic activity and acute toxicity of Sida cordifolia L. (Malva-branca). J Ethnopharmacol. 2000;72(1-2):273-277.

17. Kanth VR, Diwan PV. Analgesic, anti-inflammatory and hypoglycaemic activities of Sida cordifolia. Phytother Res. 1999;13(1):75-77.

18. Dhara AK, Suba V, Sen T, et al. Preliminary studies on the antiinflammatory and analgesic activity of the methanolic fraction of the root extract of Tragia involucrata Linn. J Ethnopharmacol. 2000;72(12):265-268.

19. Altemimi A, Lakhssassi N, Baharlouei A, et al. Phytochemicals: Extraction, Isolation, and Identification of Bioactive Compounds from Plant Extracts. Plants. 2017;6(4):42

20. Daliu P, Santini A, Novellino E. From pharmaceuticals to nutraceuticals: Bridging disease prevention and management. Expert Rev Clin Pharmacol. 2019;12(1):1-7.

21. Calvo MI. Anti-inflammatory and analgesic activity of the topical preparation of Verbena officinalis L. JEthnopharmacol. 2006;107(3):380 382

22. Winter CA, Risley EA, Nuss GW. Carregeenin-induced edema in hind paw of the rat as assay for anti-inflammatory drugs. Proc Soc Exp Biol Med. 1962;111:544-547.

23. Vasudevan M, Gunnam KK, Parle M. Antinociceptive and antiinflammatory effects of Thespesia populnea bark extract. $J$ Ethnopharmacol. 2007;109(2):264-270.

24. Dai Y, But PP, Chan YP, et al. Antipruritic and anti-inflammatory effects of aqueous extract from Si-Wu-Tang. Biol Pharm Bull. 2002; 25(9):11751178.

25. Kweifio-Okai G. Anti-inflammatory activity of a Ghanaian antiarthritic herbal preparation: I. J Ethnopharmacol. 1991;33(3):263-267.

26. Nkeh BC, Njamen D, Wandji J, et al. Anti-inflammatory and analgesic effects of Drypemolundein A, a Sesquiterpene lactone from Drypetes molunduana. Pharmaceutical Biology. 2003;41(1):26-30.

27. Dubuisson D, Dennis SG. The formalin test: a quantitative study of the analgesic effects of morphine, meperidine, and brain stem stimulation in rats and cats. Pain. 1977;4(2):161-174.

28. Gupta R, Gupta GD. Formulation Development and Evaluation of Antiinflammatory Potential of Cordia obliqua Topical Gel on Animal Model. Pharmacog J. 2017;9(6):s93-s98.

29. Panthong A, Kanjanapothi D, Taesotikul T, et al. Anti-inflammatory and antipyretic properties of Clerodendrum petasites S. Moore. Journal of Ethnopharmacology. 2003;85(1):151-156.

30. Vinegar R, Schreiber W, Hugo R. Biphasic development of carrageenan edema in rats. Journal of Pharmacology and Experimental Therapeutics. 1969;166(1):96-103.

31. Crunkhon P, Meacock SER. Mediators of the inflammation induced in the rat paw by carrageenan. British Journal of Pharmacology. 1971;42(3):392-402.

32. Collier HO, Dinneen LC, Johnson CA, et al. The abdominal constriction response and its suppression by analgesic drugs in the mouse. British Journal of Pharmacology. 1968;32(2):295-310.

33. Tjolsen A, Berge OG, Hunskaar S, et al. The formalin test: an evaluation of the method. Pain. 1992;51(1):5-17.

34. Shibata M, Ohkubo T, Takahashi $\mathrm{H}$, et al. Modified formalin test: characteristic biphasic pain response. Pain. 1989;38(3):347-352.

35. Rosland JH, Tjølsen A, Mæhle B, et al. The formalin test in mice: effect of formalin concentration. Pain. 1990;42(2):235-242.

36. Young HY, Luo YL, Cheng HY, et al. Analgesic and anti-inflammatory activities of [6]-gingerol. J Ethnopharmacol. 2005;96(1-2):207-210. 
37. Maurya S, Singh D. Quantitative analysis of total phenolic content in Adhatoda vasica Nees extracts. Int J Pharm Tech Res. 2010;2(4):24032406.

38. Mujeeb F, Bajpai P, Pathak N. Phytochemical evaluation, antimicrobial activity, and determination of bioactive components from leaves of Aegle marmelos. Biomed Res Int. 2014;2014:497606.

39. Tripathi AC, Gupta R, Saraf K. Phytochemical investigation characterisation and anticonvulsant activity of Ricinus communis seeds in mice. Natural Product Research. 2011;25(19):1881-1884.

40. Gejalakshmi S, Senthilraj R, Tanisha A. et al. Phytochemical screening and antimicrobial activity of Azadirachta indica and Plectranthus amboinicus extract. International Journal of Current Pharmaceutical Research. 2020;12(1):14-17.

41. Sundaraganapathy R, Niraimathi V, Ananda S, et al. Phytochemical studies and pharmacological screening of Sida rhombifolia Linn. Hygeia JD Med. 2013;5(1):19-22.
42. Reddy BS, Rao NR, Vijeepallam K, et al. Phytochemical, pharmacological and biological profiles of Tragia Species (Family: Euphorbiaceae). Afr J Tradit Complement Altern Med. 2017;14(3):105-112.

43. Hesham R, El- Seedi, Shgeru N. Chemistry of bioflavonoids. Indian $J$ Pharm Educ. 2007;39:172.

44. Di Carlo G, Mascolo N, Izzo AA, et al. Flavonoids: old and new aspects of a class of natural therapeutic drugs. Life Sci. 1999;65(4):337-353.

45. Rajnarayana K, Reddy MS, Chaluvadi MR, et al. Bioflavonoids classification, pharmacological, biochemical effects and therapeutic potential. Indian J Pharmacol. 2001;33(1):2-16.

46. Nunes CDR, Barreto Arantes M, Menezes de Faria Pereira S, et al. Plants as Sources of Anti-Inflammatory Agents. Molecules. 2020;25(16):3726. 